\title{
Accuracy of Different Methods for identification of Staphylococcus haemolyticus
}

\author{
Meerabai Manoharan ${ }^{1}$, Sujatha Sistla ${ }^{1}$, Pallab Ray ${ }^{2}$ \\ ${ }^{1}$ Department of Microbiology, Jawaharlal Institute of Postgraduate Medical Education and Research, Pondicherry, \\ India \\ ${ }^{2}$ Department of Medical Microbiology, Post Graduate Institute of Medical Education \& Research, Chandigarh, India
}

\begin{abstract}
Objectives: Staphylococcus haemolyticus is associated with device-related infections in immunocompromised individuals and acts as a reservoir for antibiotic resistance genes. It is also the species with the highest antibiotic resistance rates. However, identification is still difficult in most clinical laboratories. Simplified biochemical tests give variable results while newer methods such as MALDI-TOF MS and automated systems may not be readily available. Aim: To compare the performance of the simplified biochemical scheme, BD-Phoenix automated system, and PCR for nuc gene for the identification of $S$. haemolyticus with MALDI-TOF MS as the gold standard.
\end{abstract}

Methods: This study included 427 coagulase-negative staphylococci (CoNS) isolates of which 356 were identified as S. haemolyticus and 71 as other species by MALDI-TOF MS. These isolates were subjected to a simplified biochemical scheme using tests like the fermentation of maltose, sucrose, trehalose, mannose, urease, xylose, ornithine, and susceptibility to novobiocin. Conventional PCR targeting the nuc gene and BD-Phoenix were also used for identification. The accuracy of these methods was assessed in comparison with MALDI-TOF MS.

Results: The sensitivity and specificity of biochemical tests, BD- Phoenix and nuc PCR were $97.5 \%$ and $97.2 \%$ : $97.8 \%$ and 100\%: $100 \%$ and 100\% respectively. Inaccurate identification was observed for some of the isolates (2.2\% by BD- Phoenix and $2.5 \%$ by biochemical tests). These isolates were identified as $S$. haemolyticus by the other methods.

Conclusion: Identification of $S$. haemolyticus by biochemical tests and BD-Phoenix had good accuracy comparable to PCR as well as MALDI-TOF MS. This simplified biochemical scheme can be easily implemented even in laboratories with limited resources. J Microbiol Infect Dis 2021; 11(1):8-14.

Keywords: S. haemolyticus, coagulase negative staphylococci, MALDI-TOF MS, nuc gene

\section{INTRODUCTION}

Staphylococcus haemolyticus, an emerging nosocomial pathogen, is considered the second most common species of coagulase-negative Staphylococci (CoNS) [1]. Even though Staphylococcus haemolyticus was initially assumed to be harmless, it was one of the pathogens among patients with invasive and indwelling medical devices, and its role was identified as early as the 1970s [2]. This species is known to be resistant to most of the antibiotics and thus may act as a source for resistance genes to the more pathogenic species residing in the same ecological niche (e.g. S. aureus) [3]. The standard method for the identification of staphylococcal species and subspecies proposed by Kloos and Schleifer (1975) and Bannerman (2003) is based on several biochemical tests. Despite its emerging clinical significance, identification of CoNS at the species level is not routinely done in most laboratories as it is tedious and time-consuming. Hence a rapid and reliable identification method is the need of the hour [4].

The implementation of automation in microbiology laboratories had a significant impact, and matrix-assisted laser desorption ionization-time of flight mass spectrometry (MALDI-TOF MS), BD- Phoenix, Vitek 2, MicroScan Walkaway, etc. have both increased

Correspondence: Dr. Sujatha Sistla, Department of Microbiology, Jawaharlal Institute of Postgraduate Medical Education and Research, JIPMER, Pondicherry, India

Email: sujathasistla@gmail.com

Received: 05 September 2020 Accepted: 12 December 2020

Copyright (C JMID / Journal of Microbiology and Infectious Diseases 2020, All rights reserved 
the speed and accuracy with which bacteria (or any microbes) can be identified. The MALDITOF MS can be performed in a single step, and is capable of accurate identification of most of the isolates within a short time frame, while the other commercial automated systems have an accuracy of about 70-90\% [5]. Despite their popularity, these automated systems rarely make it to resource-poor settings due to high installation costs. Accurate identification of $S$. haemolyticus employed sequencing of targeted regions belonging to $16 \mathrm{~S}$ rRNA, sodA (superoxide dismutase $A$ ), tuf (elongation factor Tu), gap (glyceraldehyde-3-phosphate dehydrogenase), and rpoB genes ( $\beta$ subunit of RNA polymerase) [6]. In the present study, a new in-house developed PCR assay targeting the thermonuclease (nuc) gene was employed for the identification of $S$. haemolyticus.

The aim of the present study was to compare the performance of various tests for accurate identification of $S$. haemolyticus viz. biochemical tests, BD-Phoenix automated system, and nucPCR with MALDI-TOF MS as a gold standard method.

\section{METHODS}

\section{Study Setting}

The present study was performed in the Department of Microbiology, Jawaharlal Institute of Postgraduate Medical Education and Research, Pondicherry, India. It was approved by Institute Ethics Committee (Human Studies).

\section{Identification of CoNS}

Consecutive, non-repetitive, clinically significant isolates of coagulase-negative staphylococci (CoNS) from various specimens (blood, sterile body fluids, biopsy, and pus) sent to the Department of Microbiology from March 2016 to May 2018 were tested. The genus-level identification was carried out based on the colony morphology on $5 \%$ sheep blood agar, Gram stain, catalase test, tube coagulase test, slide coagulase test, and a commercial slide latex agglutination test (STAPHYLO LA SEIKEN, DENKA SEIKEN, Japan). The species (S. lugdunensis, S. schleiferi, S. sciuri, S. hyicus, and $S$. intermedius) negative in the tube coagulase test but positive for clumping factor were also included in this study. Species-level identification of isolates was accomplished by matrix-assisted laser desorption ionization-time of flight mass spectrometry (MALDI-TOF MS MS) (Microflex LT, Bruker Daltonik $\mathrm{GmbH}$, Bremen, Germany) performed at Postgraduate Institute of Medical Education and Research (PGIMER), Chandigarh, India following manufacturer's instructions. All the isolates confirmed as $S$. haemolyticus $(\mathrm{N}=356)$ were included in the study, while a subset of isolates belonging to other CoNS species $(\mathrm{N}=71)$ was also included giving a total number of isolates tested as 427 . These were included to test the specificity of the different methods.

\section{Biochemical tests}

The schemes of Kloos \& Schleifer (1975) and Koneman et al (1997) were used for biochemical characterization [7, 8]. While this method required more than 40 biochemical tests, a simplified two-stage procedure was followed in the present study. In stage one, fermentation of maltose, sucrose, and trehalose was tested. If all three sugars were fermented, stage two was carried out (fermentation of xylose, production of urease and ornithine decarboxylase (ODC), and susceptibility to novobiocin). Those isolates which did not ferment xylose were negative for urease and ODC and susceptible to novobiocin were identified as $S$. haemolyticus. Additional biochemical test (fermentation of mannose) was carried out only for those isolates which showed discrepant results.

\section{BD-Phoenix automated system:}

All the isolates were tested by BD-Phoenix according to the manufacturer's instructions.

\section{The nuc- PCR for identification $S$. haemolyticus:}

The nucleotide sequences of the nuc gene among all the $S$. haemolyticus isolates submitted in NCBI GenBank were aligned using CLUSTALW

(http://www.ebi.ac.uk/Tools/msa/clustalw2/) and conserved regions were identified. These conserved regions were submitted for primer design using the NCBI PRIMER-BLAST tool (https://blast.ncbi.nlm.nih.gov/Blast.cgi) using default parameters. Based on the various primers obtained, the primer pair nuc $F-5$ 'GCTGTTTTAGTGGTAGGCGT-3' and nuc R 5'-CCACACATAAGCAAGTGTCCG-3' with product size 354 bp were selected and tested for 
specificity by in silico PCR (http://insilico. ehu.es/PCR/) (supplementary data). From all the isolates DNA was extracted using a commercial Mericon DNA Bacteria plus kit (Qiagen, Germany) following the manufacturer's instructions. The PCR was then carried out in 25 $\mu \mathrm{l}$ volume in a thermal cycler (Mastercycler Nexus gradient, Eppendorf, Germany) and the reaction mix contained $2 \times$ Taq PCR Smart Mix (Origin Biolabs, India), $10 \mathrm{pmol}$ of each primer, and $5 \mu \mathrm{L}$ of sample DNA. Following were the reaction conditions - initial denaturation at $94^{\circ} \mathrm{C}$ for $4 \mathrm{~min}$; 30 cycles of denaturation at $94^{\circ} \mathrm{C}$ for $15 \mathrm{sec}$, annealing at $58^{\circ} \mathrm{C}$ for $30 \mathrm{sec}$, and extension at $72^{\circ} \mathrm{C}$ for $30 \mathrm{sec}$ and a final extension was carried out at $72^{\circ} \mathrm{C}$ for $5 \mathrm{~min}$. The amplified products were visualized by electrophoresis in $1.5 \%$ agarose gels stained with ethidium bromide. S. haemolyticus ATCC 29970 was used as a positive control in the PCR reaction. The amplification products of two isolates were sequenced using Sanger method, and confirmed as nuc genes based on sequence similarity (GenBank accession numbers MN120435 and MN120436).

\section{Statistical analysis}

For the purpose of statistical analysis, the results by MALDI-TOF MS were considered a gold standard. All the assay results were compared with this gold standard for estimating diagnostic accuracy in terms of sensitivity, specificity, positive- and negative-predictive values. The agreement between various tests was determined by kappa statistics. The statistical significance of the test results in comparison with the gold standard was analyzed by Chi-square or Fisher exact test as applicable, with $\mathrm{p}<0.05$ considered as statistically significant. All statistical analyses were performed in SPSS v19 and OpenEpi v3.01.

\section{RESULTS}

A total of 427 isolates of coagulase-negative staphylococci were tested by four different methods. Most of them (356/427; 83.4\%) were $S$. haemolyticus as confirmed by MALDI-TOF MS. The various species and subspecies identified among the 427 isolates are shown in table 1. The results obtained from biochemical tests, BD-Phoenix, and by PCR for the nuc gene were compared with MALDI-TOF MS as depicted in Table 2.

As seen from table-3, nine isolates showed aberrant results by biochemical tests (four isolates were urease positive and five were trehalose non-fermenters) and were identified as $S$. haemolyticus based on mannose nonfermentation. All the nine isolates were identified as $S$. haemolyticus by MALDI-TOF MS, BDPhoenix, and nuc-PCR. Two $S$. hominis isolates were misidentified as $S$. haemolyticus by the biochemical tests and confirmed by the other three methods. The BD-Phoenix system misidentified $S$. haemolyticus isolates (8/356; $2.2 \%$ ) as $S$. capitis (four), S. lugdunensis (one), S. pasteuri (one), and unidentified (two). BDPhoenix and biochemical tests were able to accurately identify S. haemolyticus $97 \%$ of the time. The nuc-PCR had the highest sensitivity $(100 \%)$ and specificity (100\%) followed by BDPhoenix (kappa values 1 and 0.935 respectively) when compared with MALDI-TOF MS (Figure 1).

Table 1. The species distribution of coagulasenegative staphylococci.

\begin{tabular}{lc}
\hline Species & $\begin{array}{c}\text { Total isolates } \\
\mathrm{n}=427(\%)\end{array}$ \\
\hline S. haemolyticus & $356(83.4 \%)$ \\
S. epidermidis & $28(6.6 \%)$ \\
S. hominis & $22(5.6 \%)$ \\
S. lugdunensis & $7(1.6 \%)$ \\
S. pasteuri & $5(1.2 \%)$ \\
S. intermedius & $2(0.5 \%)$ \\
S. capitis & $2(0.5 \%)$ \\
S. cohnii & $2(0.5 \%)$ \\
S. cohnii subsp urealyticum & $1(0.2 \%)$ \\
S. saprophyticus & $1(0.2 \%)$ \\
S. warneri & $1(0.2 \%)$ \\
\hline
\end{tabular}




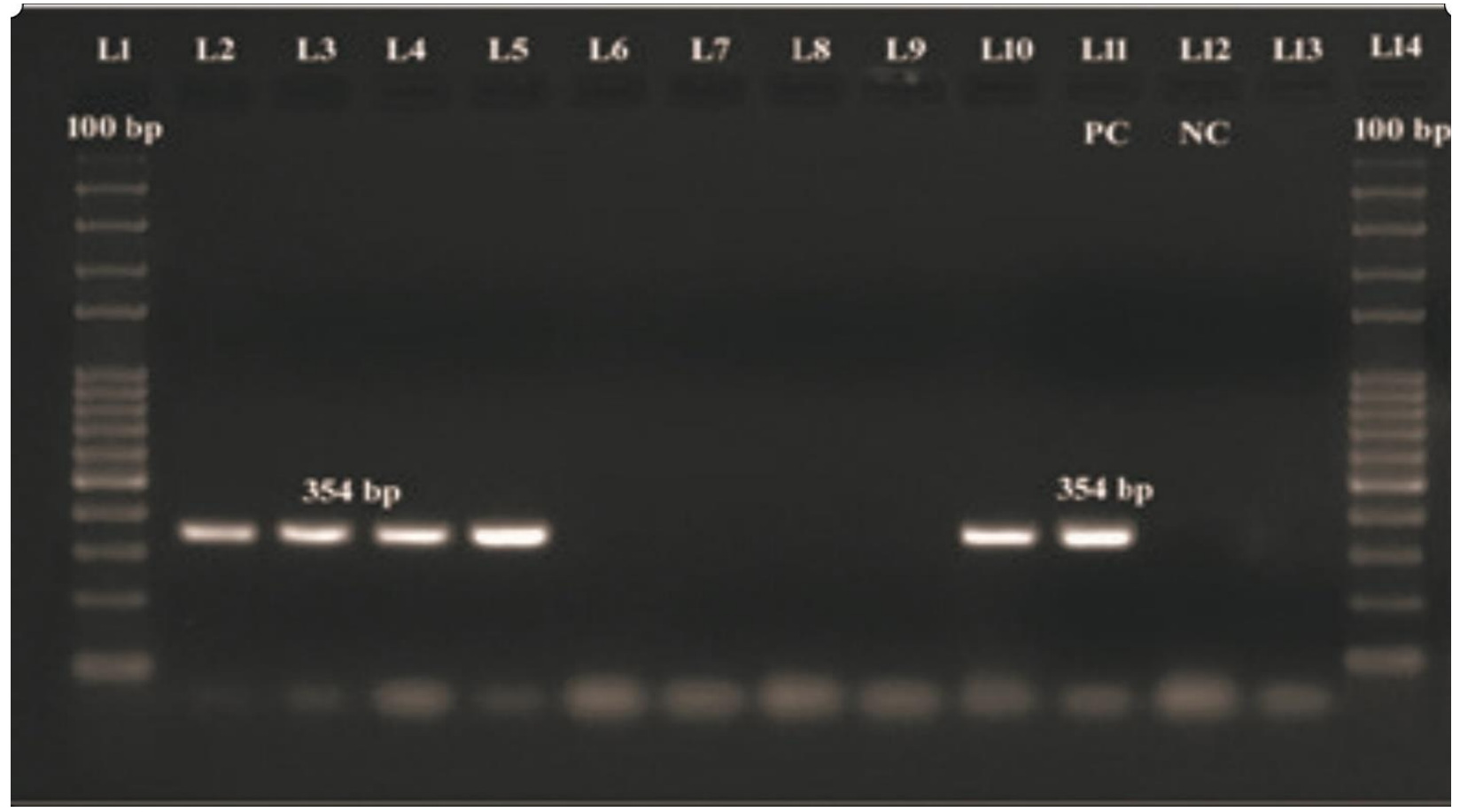

Figure 1. Gel picture of PCR reaction for nuc gene. Lane 1 (L1), L 14: 100 bp molecular marker; L2, L3, L4, L5, L10: S. haemolyticus test isolates positive for nuc gene (354bp); L6-L9, L13: Other Staphylococcus species isolates negative for nuc gene; L11- S. haemolyticus ATCC 29970; L12-Negative control (NC).

Table 2. Comparison of different tests with MALDI-TOF MS for identification of $S$. haemolyticus ( $n=427)$.

\begin{tabular}{lcccc}
\hline Test & MALDI-TOF MS $(+)$ & MALDI-TOF MS (-) & Total & $p$-value \\
\hline Biochemical tests (+) & 347 & 2 & 349 & $<0.001$ \\
Biochemical tests (-) & 9 & 69 & 78 & \\
BD-Phoenix system (+) & 348 & 0 & 348 & $<0.001$ \\
BD-Phoenix system (-) & 8 & 71 & 79 & \\
nuc-PCR (+) & 356 & 0 & 356 & $<0.001$ \\
nuc-PCR $(-)$ & 0 & 71 & 71 & \\
\hline
\end{tabular}

Table 3. Performance characteristics of different tests with MALDI-TOF MS for identification of $S$. haemolyticus ( $\mathrm{n}=$ 427).

\begin{tabular}{lccccc}
\hline Test & Sensitivity $(95 \% \mathrm{Cl})$ & Specificity $(95 \% \mathrm{Cl})$ & PPV $(95 \% \mathrm{Cl})$ & NPV $(95 \% \mathrm{Cl})$ & $\mathrm{Kappa}(95 \% \mathrm{Cl})$ \\
\hline Biochemical tests & $97.5(95.3,98.8)$ & $97.2(90.2,99.6)$ & $99.4(97.9,99.8) .88 .5(80.1,93.6)$ & $0.911(0.859-0.963)$ \\
BD-Phoenix & $97.8(95.6,98.9)$ & $100(94.9,100)$ & $100(98.9,100) 89.9(81.3,94.8)$ & $0.935(0.891-0.980)$ \\
nuc-PCR & $100(98.9,100)$ & $100(94.9,100)$ & $100(98.9 ; 100)$ & $100(94.9 ; 100)$ & $1(1.0-1.0)$ \\
\hline
\end{tabular}




\section{DISCUSSION}

Coagulase-negative staphylococci (CoNS) have so far been considered as contaminants or commensals with minimum or no clinical significance (except when isolated from medical and healthcare device-related infections). The emergence and spread of antimicrobial resistance and growing evidence suggesting the transfer of resistance elements among staphylococci (including S. aureus and MRSA) make this group of organisms important to identify and study further [9]. While a majority of the studies report $S$. epidermidis as the most common ( $75 \%)$ CoNS species among bacteremia and hospital-acquired infection (HAl) cases [10], in our experience, the majority of the CoNS isolates with clinical significance are $S$. haemolyticus followed by $S$. epidermidis [11]. $S$. hominis was found to be the most prevalent pathogen among CoNS in causing bacteremia from another center in India [12]. There are wide epidemiological gaps between the different CoNS species. Moreover, according to the Clinical and Laboratory Standards Institute (CLSI), breakpoint criteria for interpretation of the susceptibility of CoNS differ from species to species. Therefore, the identification of CoNS to the species level is important. The traditional method of identifying microorganisms to species level by biochemical tests and automated systems is time-consuming as it takes 18- 48 hours and 8-12 hours respectively. Even conventional PCR, though considered rapid, still takes 6-8 hours. On the other hand, MALDI-TOF MS provides much quicker and easier identification, within 30 minutes.

The differentiation of CoNS species requires 40 different biochemical assays proposed by Koneman et al which was long considered as the reference method [8]. Owing to the tedious procedure, many investigators have recommended only a select few biochemical assays for accurate differentiation of CoNS species. Sah et al in 2018 employed five tests mannitol, maltose, mannose, trehalose fermentation tests, and novobiocin susceptibility for identification of $S$. epidermidis and found them to be highly specific for identification of $S$. epidermidis, confirmed by $16 \mathrm{~S}$ rRNA sequencing [13]. Cunha et al employed a two-stage biochemical method where the first stage identified $S$. haemolyticus group (includes $S$. lugdunensis, S. saprophyticus, and S. warneri along with $S$. haemolyticus). Isolates from this group were then tested for stage two biochemicals (fructose, urease, ornithine decarboxylase, and resistance to novobiocin) which can confirm the presence of $S$. haemolyticus. This two-stage assay was 100\% in agreement with the reference method (Kloos \& Schleifer (1975) and Bannerman (2003) [4]. Many investigators have employed this strategy for species-level identification of CoNS [14-16].

In our study, we employed three biochemical tests - fermentation of sucrose, trehalose, and maltose as $S$. haemolyticus is known to be a fermenter of these three sugars. However, Cunha et al reported other species of staphylococci that were also fermenters of these sugars - $S$. saprophyticus, $S$. warneri, $S$. lugdunensis, $S$. xylosus, $S$. hominis, and $S$. simulans. While $S$. hominis, $S$. simulans, and $S$. xylosus were actually confirmed using a few more biochemical tests like hemolysis, xylose, mannitol, and anaerobic growth on thioglycollate [4], in the present study, xylose fermentation was targeted. The fermentation of thioglycollate requires anaerobic growth conditions, and the objective of the study was to make the most simple and effective differentiation method and was therefore excluded from the present study. S. saprophyticus, S. warneri, S. lugdunensis can be differentiated from $S$. haemolyticus by targeting urease production, ornithine decarboxylase, and resistance to novobiocin, and these three tests were included in this study.

Our protocol which used seven tests was thus shorter than that of Cunha et al where ten tests were performed in two stages [4]. Although the protocol by Goyal et al used only six tests (excluding coagulase test) for confirmation of $S$. haemolyticus among CoNS, all the tested isolates in the stage one were urease negative [17]. Our protocol aims to resolve inconsistencies that may arise during the differentiation of $S$. haemolyticus by exploiting the fermentation of mannose.

The slight inaccuracy led to a very small decrease in specificity (97.2\%). In contrast with the studies by Cunha et al and Goyal et al $[4,17]$ we used a significantly larger number of isolates and compared our results with a very accurate 
MALDI-TOF MS (Cunha et al used reference biochemical tests as the gold standard, while no gold standard was considered in the study by Goyal et al). The former two studies had a total of eight and $14 \mathrm{~S}$. haemolyticus isolates respectively, while the number of $S$. haemolyticus isolates in the present study was 356. This large increase in the number of isolates tested can give a few inaccurate results purely by chance. However, our protocol minimized this chance error significantly and the specificity of $97.2 \%$ can still be considered optimum especially for screening assays like testing for biochemicals.

The Microflex system was highly accurate $(100 \%)$ in a study by Spanu et al when compared with the rpoB gene sequencing method [18]. Similar to this Trevisoli et al. and Loonen et al employed the tuf gene sequencing and MALDI-TOF MS and reported 100\% accuracy in identifying $S$. haemolyticus $[19,20]$. Tomazi et al. compared MALDI-TOF MS with PCR-RFLP of the groEL gene and found only $80 \%(4 / 5)$ of the $S$. haemolyticus was accurately identified by MALDI-TOF MS [21]. In the present study $356(83.4 \%)$ isolates were confirmed as $S$. haemolyticus while $71 \quad(16.6 \%)$ isolates belonged to other species and $S$. epidermidis $(6.6 \%)$ followed by $S$. hominis $(5.6 \%)$ were the most common among other species of CoNS. The confidence with which these species were identified exceeded $98 \%$, thereby there was no scope of aberrant interpretations by the system.

The BD-Phoenix employs a series of 45 assays to identify microbial species. In the present study, it fared slightly lower by identifying $346 / 356$ isolates giving overall sensitivity of $97.8 \%$ and $100 \%$ specificity. Two isolates were not resolved at the species level by BD-Phoenix (S. haemolyticus / $S$. lugdunensis) requiring confirmation by an additional biochemical test (ornithine decarboxylase). Layer et al. tested BD-Phoenix on 30 isolates of $S$. haemolyticus and found it to be less sensitive (90\%) [22]. Based on a meta-analysis by Chatzigeorgiou et al the accuracy of BD-Phoenix was $88.4 \%$ for species-level identification of CoNS, thereby demonstrating that misidentification may be more common while using BD- Phoenix [23]. Interestingly, the nine isolates misidentified in the first two steps of biochemical assays were accurately identified as $S$. haemolyticus by BDPhoenix.

Various genes were targeted for accurate differentiation of CoNS species. A multiplex PCR has been developed by Hirotaki et al to aid in the differentiation of a few species of staphylococci including $S$. aureus and $S$. haemolyticus. In the study nuc genes of 24 staphylococcal species were selected and primers were designed that were specific to the nuc gene of each species. Based on the primer sequences, accurate identification of $S$. haemolyticus was achieved, and the overall sensitivity and specificity of this multiplex PCR was $100 \%$ when compared with hsp60 gene sequences [24]. In the present study, however, primers specific to nuc genes of $S$. haemolyticus were designed and selected. Among the 427 isolates tested, the PCR was positive for all the 356 S. haemolyticus isolates and did not yield any false-positive results. Thus nuc-PCR method was $100 \%$ sensitive and specific and in perfect agreement with MALDI-TOF MS.

\section{Conclusions}

The nuc-PCR designed in this study was found to be the best method for the identification of $S$. haemolyticus from a group of CoNS. We developed and evaluated a simple affordable biochemical-based method and found it to be highly sensitive and specific for the identification of $S$. haemolyticus. While a few isolates may show aberrant results, combined together, they are highly accurate. Based on these findings and the low cost involved, it is recommended to use biochemical tests for the identification of $S$. haemolyticus especially in laboratories with limited resources.

\section{ACKNOWLEDGMENTS}

Declaration of Conflicting Interests: The authors declare that they have no conflict of interest.

Funding: This research work was financially supported by JIPMER Intramural Research fund (JIPMER, Puducherry) and Indian Council of Medical Research Antimicrobial Resistance Surveillance Project (ICMR_AMRSN).

\section{REFERENCES}

1. Bouchami $\mathrm{O}$, de Lencastre $\mathrm{H}$, Miragaia $\mathrm{M}$. Impact of insertion sequences and recombination on the population structure of Staphylococcus 
haemolyticus. PloS One. 2016; 11(6):e0156653e0156653.

2. Czekaj T, Ciszewski M, Szewczyk EM. Staphylococcus haemolyticus - an emerging threat in the twilight of the antibiotics age. Microbiology 2015; 161(11):2061-8.

3. Cavanagh JP, Hjerde E, Holden MTG, et al. Whole-genome sequencing reveals clonal expansion of multi-resistant Staphylococcus haemolyticus in European hospitals. J Antimicrob Chemother 2014; 69(11):2920-7.

4. Cunha M de LR, Sinzato YK, Silveira LV. Comparison of methods for the identification of coagulase-negative staphylococci. Mem Inst Oswaldo Cruz 2004; 99(8):855-60.

5. Becker K, Heilmann C, Peters G. Coagulasenegative Staphylococci. Clin Microbiol Rev 2014; 27(4):870-926.

6. Kosecka-Strojek M, Sabat AJ, Akkerboom V, et al. Development and Validation of a Reference Data Set for Assigning Staphylococcus Species Based on Next-Generation Sequencing of the 16S-23S rRNA Region. Front Cell Infect Microbiol 2019; 9:278-278.

7. Kloos WE, Schleifer KH. Simplified scheme for routine identification of human Staphylococcus species. J Clin Microbiol 1975; 1(1):82-8.

8. Koneman E.W, Allen S.D, Janda W.M, et al. Staphylococci and related organisms. Color Atlas and Textbook of Diagnostic Microbiology 1997; 539-576.

9. Rossi CC, Pereira MF, Giambiagi-deMarval M. Underrated Staphylococcus species and their role in antimicrobial resistance spreading. Genet $\mathrm{Mol}$ Biol 2020; 43(1 suppl 2):e20190065.

10. Kleinschmidt S, Huygens F, Faoagali J, et al. Staphylococcus epidermidis as a cause of bacteremia. Future Microbiol 2015; 10(11):1859-79.

11. Manoharan M, Sistla S, Ray P. Prevalence and molecular determinants of antimicrobial resistance in clinical isolates of Staphylococcus haemolyticus from India. Microb Drug Resist [Internet] 2020 Mar 19 [cited 2020 Jul 14].

12. Gautam V, Sethuraman N, Kaur R, et al. Changing epidemiology of coagulase-negative staphylococci in normal flora of skin. Indian $\mathrm{J}$ Med Microbiol 2017; 35(2):277.

13. Sah S, Bordoloi P, Vijaya D, et al. Simple and economical method for identification and speciation of Staphylococcus epidermidis and other coagulase negative Staphylococci and its validation by molecular methods. J Microbiol Methods 2018; 149:106-19.

14. Pinheiro L, Brito $\mathrm{Cl}$, Pereira VC, et al. Susceptibility profile of Staphylococcus epidermidis and Staphylococcus haemolyticus isolated from Blood cultures to vancomycin and novel antimicrobial drugs over a period of 12 years. Microb Drug Resist 2016; 22(4):283-93.
15. de Oliveira A, Cataneli Pereira V, Pinheiro L, et al. Antimicrobial resistance profile of planktonic and biofilm cells of Staphylococcus aureus and coagulasenegative staphylococci. Int J Mol Sci 2016; 17(9):1423.

16. de Freitas Guimarães F, Nóbrega DB, RichiniPereira VB, et al. Enterotoxin genes in coagulasenegative and coagulase-positive staphylococci isolated from bovine milk. J Dairy Sci 2013; 96(5):2866-72.

17. Goyal R, Singh NP, Kumar A, et al. Simple and economical method for speciation and resistotyping of clinically significant coagulase negative staphylococci. Indian J Med Microbiol 2006; 24(3):201-4.

18. Spanu T, De Carolis E, Fiori B, et al. Evaluation of matrix-assisted laser desorption ionization-time-offlight mass spectrometry in comparison to rpoB gene sequencing for species identification of bloodstream infection staphylococcal isolates. Clin Microbiol Infect 2011; 17(1):44-9.

19. Trevisoli LE, Bail L, Rodrigues LS, et al. Matrixassisted laser desorption ionization-time of flight: a promising alternative method of identifying the major coagulase-negative staphylococci species. Rev Soc Bras Med Trop 2018; 51(1):85-7.

20. Loonen AJM, Jansz AR, Bergland JNB, et al. Comparative study using phenotypic, genotypic, and proteomics methods for identification of coagulasenegative staphylococci. J Clin Microbiol 2012; 50(4):1437-9.

21. Tomazi T, Gonçalves JL, Barreiro JR, et al. Identification of coagulase-negative staphylococci from bovine intramammary infection by Matrixassisted laser desorption ionization-time of flight mass spectrometry. J Clin Microbiol 2014; 52(5):165863.

22. Layer F, Ghebremedhin B, Moder K-A, et al. Comparative study using various methods for identification of Staphylococcus species in clinical specimens. J Clin Microbiol 2006; 44(8):2824-30.

23. Chatzigeorgiou K-S, Sergentanis TN, Tsiodras $\mathrm{S}$, et al. Phoenix 100 versus Vitek 2 in the identification of gram-positive and gram-negative bacteria: a comprehensive meta-analysis. J Clin Microbiol 2011; 49(9):3284-91.

24. Hirotaki S, Sasaki T, Kuwahara-Arai K, et al. Rapid and accurate identification of humanassociated staphylococci by use of multiplex PCR. J Clin Microbiol 2011; 49(10):3627-31. 